\title{
Serum Receptor Activator of Nuclear Factor- $\kappa \beta$ Ligand and Osteoprotegerin Levels and Ratio in Correlation with Bone Mineral Density
}

\author{
Fauqa Arinil Aulia ${ }^{1}$, Sri Lestari Utami ${ }^{2}$, Leonita Anniwati ${ }^{1}$, Sony Wibisono Mudjanarko ${ }^{3}$, Ferdy \\ Royland Marpaung ${ }^{1}$
}

${ }^{1}$ Department of Clinical Pathology, Faculty of Medicine, Airlangga University/Dr. Soetomo Hospital, Surabaya, Indonesia. E-mail: fauqa.arinil.aulia@gmail.com

${ }^{2}$ Department of Biomedicine, Faculty of Medicine, Wijaya Kusuma University, Surabaya, Indonesia

${ }^{3}$ Department of Internal Medicine, Faculty of Medicine, Airlangga University/Dr. Soetomo Hospital, Surabaya, Indonesia

\begin{abstract}
Osteoporosis is a disorder represented by manifestations of low bone mass, decreased bone tissue, and disrupted bone microarchitecture. The diagnosis of osteoporosis so far has been based on fracture manifestations after minimal trauma or by detecting low Bone Mineral Density (BMD). Measurement of Receptor Activator of Nuclear Factor-K $\beta$ Ligand (RANKL) and Osteoprotegerin (OPG) levels has opened the discourse of a more specific assessment of osteoblast and osteoclast regulation. The RANKL/OPG ratio can represent resorption and bone formation more significantly when correlated with BMD features. This study aimed to analyze the correlation between serum RANKL and OPG levels and ratio with BMD. A total of 58 post-menopausal females from 13 elderly in Integrated Community Health Care Surabaya and Sidoarjo were enrolled. Data were collected by recording age, onset of menarche, onset of menopause, and Body Mass Index (BMI). Serum RANKL and OPG levels were evaluated using sandwich ELISA from Elabscience ${ }^{\circ}$. The RANKL/OPG ratio was obtained from the ratio between measured RANKL and OPG levels in serum. The proximal femur and lumbar spine BMDs were measured using Hologic ${ }^{\circledR}$ Discovery ${ }^{\mathrm{TM}}$ QDR $^{\mathrm{TM}}$ Dual-Energy X-ray Absorptiometry (DEXA). Pearson's correlation test in this study showed no significant correlation between BMD and RANKL levels (lumbar: $p=0.203$; hip: $p=0.283$ ). The insignificant result was also shown in the correlation between BMD and OPG levels (lumbar: $p=0.412 ;$ hip: $p=0.617$ ). A significant result between lumbar $B M D$ and RANKL/OPG ratio was only found in the osteopenia subjects $(p=0.001)$. The RANKL/OPG ratio had a significant correlation only with osteopenia-BMD in post-menopausal females. Therefore, it could be used as supporting data in osteoporosis screening.
\end{abstract}

Keywords: RANKL, osteoprotegerin, bone mineral density, osteoporosis, post-menopausal females

\section{INTRODUCTION}

Osteoporosis is a disorder represented by a manifestation of low bone mass. This disease is also characterized by decreased bone tissue and disrupted bone microarchitecture. This can lead to an increase in fracture risk. ${ }^{1}$ Osteoporosis and fractures associated with this disease are important causes of mortality and morbidity throughout the world. It was predicted that there would be an increase from $5.3 \%$ of the Asia elderly osteoporosis population in 1995 to $9.3 \%$ in $2025 .{ }^{2}$ The Indonesian Osteoporosis Association in 2007 stated that the osteoporosis patients proportion aged over 50 years in the population consisted of $32.3 \%$ female and $28.8 \%$ male. Hospital Information System Data (SIRS) in 2010 showed that the upper thigh fractures incidence generated by osteoporosis was \pm 200 out of 100,000 cases at around 40 years. ${ }^{3}$

The diagnosis of osteoporosis is established by finding fracture manifestations after minimal trauma or by discovering low Bone Mineral Density (BMD). There are several imaging modalities, but Dual Energy X-ray Absorption (DEXA) is the most recommended method. The selection of osteoporosis treatment based on the Pharmaceutical Benefits Scheme (PBS) requires confirmation of fracture due to minimal trauma or low BMD. The weakness of DEXA in describing osteoporosis can be caused by a large number of artifacts (including previous fractures), pathological processes in the spine, extrinsic artifacts, and obesity. ${ }^{4}$

The destruction rate and bone cell production could be assessed by evaluating the activity of osteoblastic enzymes, osteoclastic enzymes, and 
bone matrix components in the blood or urine stream. RANKL (as resorption marker) is classified as a new biochemical marker for bone metabolism. Osteoprotegerin (as formation marker) is also classified as a new biochemical marker of bone metabolism. RANKL and OPG can represent the osteoblasts and osteoclasts regulation more specifically. RANKL and OPG have lower biological variation in healthy populations in terms of age, gender, sampling time, menstrual cycle, and physical activity. $^{5}$

The C-terminal Telopeptide (CTX) and osteocalcin biochemical concentration are known to be affected by a diet mediated by GLP2 (glucagon-like peptide-2). This parameter is also influenced by age, gender, weight, race, pregnancy and breastfeeding history, diet, daily activities and exercise, menstrual cycle, previous history of fractures, previous history of bone metabolic diseases, liver function, and kidney function. ${ }^{5}$ RANKL and OPG have lower biological variations in the population of healthy people in terms of age, gender, sampling time, menstrual cycle, and physical activity. $^{6-8}$

The OPG regulation has shown effective results in preventing bone resorption. The RANKL measurement provides an understanding of the fractured status through the osteoporosis mechanism. Pre-clinical data indicate that high RANKL level, low OPG level, and the increase in the RANKL/OPG ratio are related to the high turnover causing loss of bone mass. The RANKL/OPG ratio can represent bone resorption and formation activities more significantly than RANKL and OPG levels when correlated with BMD images. ${ }^{5}$ This research aimed to analyze RANKL and OPG levels and ratios in correlation with BMD.

\section{METHODS}

This cross-sectional research was organized with an observational prevalence study design. This research was performed in 2019 at the Central Laboratory of Clinical Pathology Unit Dr. Soetomo Academic Hospital Surabaya, Indonesia. Subjects were 58 post-menopausal females who came to Integrated Community Health Care for the elderly in 12 villages of Sidoarjo, Indonesia, and one village of Surabaya, Indonesia. Characteristics of the study subjects were post-menopausal females who were willing to take part in the study and signed informed consent. Females in perimenopausal or post-menopausal period with osteoarthritis, rheumatoid arthritis, breast cancer that has metastasized to the bones, and diabetes mellitus were not included in this study.

Body Mass Index (BMI) is body weight in kilograms divided by height in meters squared and expressed in $\mathrm{kg} / \mathrm{m}^{2}$. It is a measure for indicating nutritional status in adults with GEA Medical ZT 120 Manual Weight and Height Scale. ${ }^{9}$ Bone mineral density measurements were carried out by evaluating the lumbar spine and femoral neck using Dual-Energy X-ray Absorptiometry (DEXA) (Hologic $₫$ DiscoveryTM). Bone mineral density is further classified according to World Health Organization (WHO) criteria into: osteoporosis if T-score $<-2.5(n=38)$, osteopenia if $-2.5<$ T-score $<-1(n=18)$, and normal if T-score $>-1(n=2) .{ }^{10}$ Total $3 \mathrm{~mL}$ of venous blood was taken and collected in a tube without anticoagulants. The serum was immediately separated by a $2500 \mathrm{rpm}$ centrifugation process for 10-15 minutes. The serum obtained was then stored in separate aliquots and frozen at $-80^{\circ} \mathrm{C}$. RANKL and OPG levels were determined using a polyclonal antibody-based sandwich ELISA from Elabscience ${ }^{\circledR}$ Biotechnology Inc., the USA, with a coefficient of variation $<10 \%$. The ELISA microplate provided in the kit has previously been coated with antibodies that are specific to human RANKL and OPG. A standard or sample was put to the well of the ELISA microplate and mixed with specific antibodies. Biotin-coated antibodies specific to human RANKL and OPG and the Avidin-Horseradish Peroxidase (HRP) conjugate were put to each microplate and then incubated. The free component was then rinsed. The substrate solution was put to each well. Blue color only appeared in wells containing human RANKL and OPG, detection antibodies coated by biotin, and the Avidin-HRP conjugate. The enzyme-substrate reaction was stopped by adding the stop solution until changes into yellow color were observed. Optical Density (OD) was measured by a spectrophotometer with a $450 \pm 2 \mathrm{~nm}$ wavelength. Optical density values were directly compared with measured human RANKL and OPG concentration. Measurement of human RANKL levels was carried out by comparing the OD of the sample with the OD of the standard curve. ${ }^{11,12}$ The RANKL/OPG ratio was obtained by dividing the measured RANKL level by the measured OPG level in serum.

This research was approved by the Medical Research Ethics Committee at the Faculty of Medicine, Airlangga University, Surabaya, Indonesia (No.149/EC/KEPK/FKUA/2019). The Kolmogorov-Smirnov, one sample test, showed normally distributed data $(P>0.05)$. Spearman's rank 
test was used in this study to determine the correlation between the nominal-scale data of the RANKL/OPG ratio and the ordinal-scale data of BMD. A value of $P<0.05$ was determined significant. Statistical analysis was carried out using IBM SPSS statistics inc.ver.22.

\section{RESULTS AND DISCUSSION}

There were 58 post-menopausal females age 50-72 years. The mean age of subjects in this study $( \pm 2 S D)$ was $60.84 \pm 5.31$ years. The average age of menopausal subjects was 48.65 years. The mean BMD T-scores $( \pm 2 S D)$ of the lumbar spine and femoral neck were $-2.68 \pm 1.03$ and $-1.92 \pm 0.76$, respectively. Bone mineral density criteria grouping found in this study based on the T-score was osteoporosis (65.5\%), osteopenia (31\%), and normal (3.5\%). The mean value of ( $\pm 2 \mathrm{SD})$ RANKL level, OPG level, and RANKL/OPG ratio were $1166,89 \pm 416,12$ $\mathrm{pg} / \mathrm{mL}, \quad 8.97 \pm 2.15 \mathrm{ng} / \mathrm{mL}$, and 140.32 \pm 71.09 , respectively. The range of RANKL and OPG levels measured in 566 healthy people aged $18-75$ years in a row was $105-4468 \mathrm{pg} / \mathrm{mL}$ and $674-4929 \mathrm{pg} / \mathrm{mL}$, respectively. ${ }^{13}$ The mean onset of menarche age, menopause age, BMI, number of pregnancies, number of live children, BMD T-score of lumbar spine, BMD T-score of femoral neck, RANKL levels in serum, OPG levels in serum, and RANKL/OPG ratios can be seen in Table 1.

Table 1. Characteristics of research subjects

\begin{tabular}{lcc}
\hline \multicolumn{1}{c}{ Categories (n=58) } & Mean & SD \\
\hline Age (years) & 60.84 & 5.31 \\
The onset of menarche (years) & 14.09 & 2.03 \\
The onset of menopause (years) & 48.65 & 4.95 \\
BMI (kg/m ${ }^{2}$ ) & 25.59 & 4.06 \\
Pregnancy (times) & 4 & 1 \\
Living children (children) & 3 & 1 \\
T-score of the femoral neck & -1.92 & 0.76 \\
T-score of the lumbar spine & -2.68 & 1.03 \\
RANKL (pg/mL) & 1166,89 & 416,12 \\
OPG (ng/mL) & 8.97 & 2.15 \\
RANKL/OPG ratio & 140,32 & 71.09 \\
\hline
\end{tabular}

Bone mineral density measurements in the lumbar region were carried out on 58 study subjects. BMD measurements in the hip region were carried out on 57 study subjects. Only one study subject did not measure BMD in the hip region because there was osteoporosis with severe bone damage that was difficult to evaluate. The results of BMD measurements in the lumbar and hip regions showed that two study subjects had normal BMD, 18 study subjects had osteopenia BMD, and 38 people had osteoporotic BMD. The results data of BMD measurements can be seen in Table 2 .

Tabel 2. Results of BMD measurement

\begin{tabular}{lcc}
\hline BMD Criteria & $\mathbf{n}$ & \% \\
\hline Normal & 2 & 3.5 \\
Osteopenia & 18 & 31.0 \\
Osteoporosis & 38 & 65.5 \\
Total & 58 & 100 \\
\hline
\end{tabular}

The Pearson correlation test showed that BMI was highly significant negative correlated $(P=-0.374$, $r=0.004)$ and RANKL/OPG ratio ( $P=0.320, r=0.014)$. Bone mineral density was also significantly correlated with the T-scores of lumbar spine BMD $(P=0.381, r=0.003)$ and $T$-scores of femoral neck BMD ( $P=0.447, r=0.000)$. This statistical test was also performed to analyze the correlation between the lumbar spine and femoral neck BMD T-scores with serum RANKL levels, serum OPG levels, and RANKL/OPG ratios. The results indicated a positive correlation between the T-score of lumbar spine $B M D$ and T-score of femoral neck BMD with RANKL levels, although not significant $(p=0.189, r=0.155$; $p=0.220, r=0.100$; respectively). T-scores of lumbar spine BMD and T-scores of femoral neck BMD were negatively correlated with OPG levels, although not significant $(p=-0.003, r=0.980 ; p=-0.113, r=0.401$; respectively). T-scores of the lumbar spine and femoral neck were positively correlated with the RANKL/OPG ratio, although not significant $(p=0.100$, $r=0.456 ; p=0.194, r=0.147$; respectively). Each correlation coefficient and $p$-value can be seen in Table 3.

Table 3. Bivariate correlation of BMI with lumbar BMD, femur BMD, RANKL, OPG, and RANKL/OPG ratio

\begin{tabular}{lccc}
\hline \multirow{2}{*}{ Bivariate Correlation } & \multicolumn{2}{c}{ BMI } & \multirow{2}{*}{ N } \\
\cline { 2 - 3 } & Pearson Correlation & Sig. (2-tailed) & 57 \\
\hline Lumbar BMD & 0.447 & $\mathbf{0 . 0 0 0}$ & 58 \\
Femur BMD & 0.381 & $\mathbf{0 . 0 0 3}$ & 58 \\
RANKL level & 0.084 & 0.530 & 58 \\
OPG level & -0.374 & $\mathbf{0 . 0 0 4}$ & 58 \\
RANKL/OPG ratio & 0.320 & $\mathbf{0 . 0 1 4}$ & \\
\hline
\end{tabular}


Correlations between each BMD group based on WHO criteria with RANKL levels, OPG levels, and RANKL/OPG ratios were also interpreted (Table 4). Correlation between normal BMD groups and RANKL/OPG ratios could not be analyzed because there were only two research subjects in the group. There was a significant positive correlation between the osteopenia BMD group $(n=18)$ and the RANKL/OPG ratio $(r=0.614, p=0.007)$. There was a significant positive correlation between osteoporosis BMD group $(n=38)$ and RANKL levels $(r=0.322, p=0.049)$. The boxplot diagram of the BMD correlation between osteopenia and osteoporosis with RANKL levels (Figure 1), OPG levels (Figure 2), and RANKL/OPG ratios (Figure 3) were listed below.

The research subjects consisted of 58 post-menopausal females (average age $=60.84$ years). The average age of menopausal females in this study was close to research in Indonesia in 2000, which showed the average age of 47 years, and in 2010, which showed the average age of 45 years. A total of 4 million females in the United Kingdom with the age of 45-51 years began the transition to menopause with the average age of menopause onset at the age of 45-55 years. Australian females begin menopause with a mean age of $51-52$ years. ${ }^{14}$

Table 4. The correlation of osteopenic and osteoporotic BMD T-score with RANKL, OPG, and RANKL/OPG ratio

\begin{tabular}{lcccc}
\hline & \multicolumn{2}{c}{ Osteopenic BMD $(\mathbf{n = 1 8})$} & \multicolumn{2}{c}{ Osteoporotic BMD $(\mathbf{n = 3 8})$} \\
\cline { 2 - 5 } & $\mathbf{r}$ & $\mathbf{P}$ & $\mathbf{r}$ & $\mathbf{P}$ \\
\hline RANKL & 0.428 & 0.076 & 0.322 & $\mathbf{0 . 0 4 9}$ \\
OPG & -0.424 & 0.079 & 0.008 & 0.964 \\
RANKL/OPG & 0.614 & $\mathbf{0 . 0 0 7}$ & 0.211 & 0.203 \\
\hline
\end{tabular}
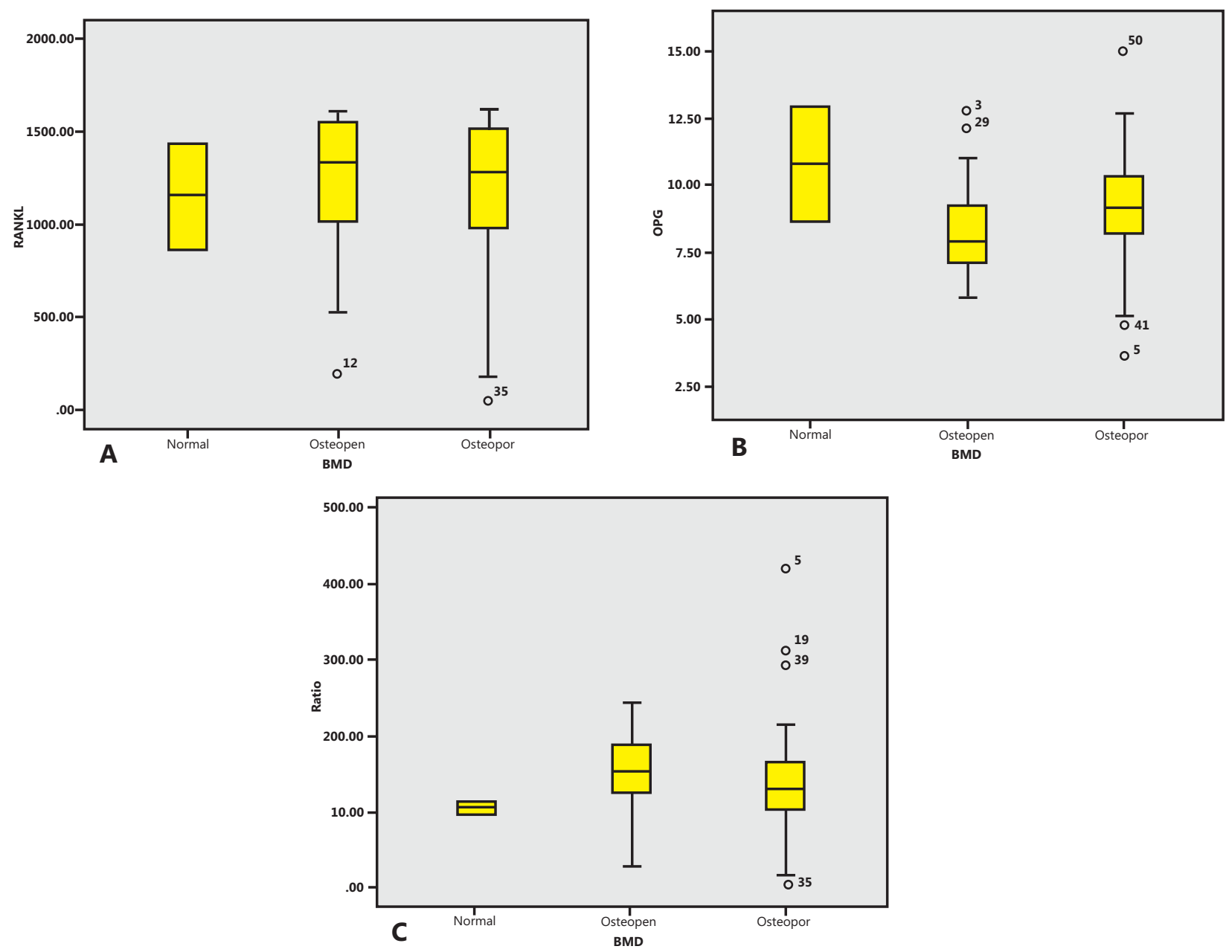

Figure 1. The Boxplot diagram of a correlation between (a) normal, osteopenic, and osteoporotic BMD with RANKL level, (b) normal, osteopenic, and osteoporotic BMD with OPG level, (c) normal, osteopenic, and osteoporotic BMD with RANKL/OPG ratio 
The average age of menopausal females in Indian is 46.2 years, lower than in western countries, which is 51 years. ${ }^{15}$ Differences in the average age of menopause could be caused by differences in the menstrual cycle. Long menstrual cycles will hinder the process of ovum maturation. Short menstrual cycles will accelerate the process of ovum maturation, leading to a faster process of ovum reduction..$^{14}$ The onset of menopause also varies according to the race and ethnicity of each female. This could be influenced by demographic and lifestyle factors. The onset of menopause for a female is positively related to the onset of menopause that occurs in the mother. Other factors that influence the onset of menopause that can be modified include BMI, diet (number of calories and alcohol consumption), and passive exposure to cigarettes. ${ }^{16}$

This study showed that OPG levels were influenced by BMI with a negative correlation. A higher BMI led to a lower OPG level in the blood. The RANKL/OPG ratio will also be affected, along with changes in OPG levels that are affected by BMI. Previous studies in Greece also found a negative correlation between serum OPG levels with BMI (multivariate analysis) and age (univariate analysis). ${ }^{17}$ Overweight will reduce the intensity of physical activity, thereby giving a negative effect on the bones by reducing the production of OPG. Overweight also correlates with lower bone mass, leading to the poor response of osteoclasts to OPG. ${ }^{18}$ Decreased OPG production by osteoblasts due to obesity can be mediated by leptin. ${ }^{19}$

Bone mineral density in this study influenced the BMD images evaluated in two sites: the lumbar spine and femoral neck. Bone mineral density can be a good indicator of the BMD images obtained from DEXA scanning. Similar results also show a significant correlation between BMI and BMD of the femoral neck and lumbar spine in populations in Iran. ${ }^{20-23}$ Low body weight is closely related to osteoporosis. ${ }^{24}$ Lower BMI and loss of bone strength are associated with a bone mass reduction in all body parts..$^{25}$ The $\mathrm{BMI}$ and $\mathrm{BMD}$ have a significant positive correlation to the pelvis, lumbar spine, and femoral neck. ${ }^{26}$ Soft tissue can contribute to refract BMD values measured by DEXA in subjects with osteopenia and osteoporosis. ${ }^{27}$

The high percentage of osteoporosis (66\%) from BMD examination results in this study was different from the estimated osteoporosis incidence rate in previous studies. Osteoporosis is expected to occur in $13-18 \%$ of the United States females aged $>50$ years, and osteopenia manifestations are found in $37-50 \%$. Decreased bone mass increases in female a few years after menopause, whereas in males, it occurs slower. Both gender have the same rate of bone mass decrease at 60-70 years. Fractures in old age are associated with fragile bone tissue. The standard measurement of bone fragility can be performed with a densitometer. The risk of fractures increases by 1.5-3 times higher in decreasing BMD for every one Standard Deviation (SD). ${ }^{28}$ The difference in osteoporosis incidence in this study with the other studies can be caused by modifiable and non-modifiable risk factors. Modifiable risk factors include sex hormones, anorexia nervosa, diet (calcium and vitamin D), consumption of long-term corticosteroids, lifestyle, smoking, alcohol and coffee consumption, and hyperparathyroidism. Non-modifiable risk factors include age, gender, ethnicity, BMI, and history of family illness. ${ }^{29}$ Bone mineral density is the factor that most influences the BMD image through OPG metabolism.

As many as $66 \%$ of study subjects with osteoporosis in this study were at risk of suffering from a fracture as previous studies results. The estimated prevalence of forearm, pelvis, and vertebral fractures in white Americans aged 50 years or more is around $40 \%$. Fractures due to osteoporosis are associated with pain, disability, and increased mortality. Vertebral fractures can cause long-term morbidity. Mortality caused by hip fractures in osteoporosis females increases four times more than males. ${ }^{30}$

The increase in serum RANKL in this study can significantly represent the severity of bone mass reduction based on BMD only in the osteoporotic group. The same results were also obtained from studies in the post-menopausal female population in Iran, which showed a significant positive correlation between BMD and RANKL. ${ }^{23}$ The osteopenic group in this research had a non-significant correlation with BMD and RANKL levels. The same results from a study in the post-menopausal female population in China, suggesting that there was no correlation between BMD and RANKL levels. ${ }^{24}$

The decrease in serum OPG in this study did not significantly represent the severity of bone mass reduction based on BMD. This was consistent with studies in China post-menopausal female populations, which showed that OPG levels were not significantly related to BMD. ${ }^{31}$ Another cause of insignificant OPG levels with BMD is an increase in OPG levels into a defense response mechanism of the paracrine system by bone cells to inhibit resorption. Increased levels of OPG also reflect bone remodeling acceleration. ${ }^{25}$ Research on the Iran post-menopausal female population showed 
different results from this study, in which a significant negative correlation between OPG levels and BMD was found. ${ }^{23}$

The RANKL/OPG ratio in this research can specifically describe the significant severity of bone mass reduction based on BMD only in the osteopenic group. This was consistent with research performed on a healthy population of Iran post-menopausal females. The RANKL/OPG system correlates with $B M D$ if there is no systemic inflammation that affects bone mass. ${ }^{23}$ The presence of chronic inflammation or autoimmune processes can affect bone metabolism in the RANKL/OPG pathway. ${ }^{32}$ The bone resorption process mediated by the RANKL/OPG system is also influenced by 1,25 -dihydroxy vitamin $D$, parathyroid hormone, and interleukin $-1 .^{33}$ The highest RANKL/OPG ratio is found in the mesenchymal stromal cells in the bone marrow. The RANKL/OPG ratio can decrease with osteoblastic cell differentiation, which can cause increased osteoclastogenesis. ${ }^{25}$

\section{CONCLUSION AND SUGGESTION}

The RANKL/OPG ratio had a significant correlation only with osteopenia-BMD in post-menopausal females. Therefore, it could be used as supporting data in osteoporosis screening. The RANKL/OPG ratio could represent the severity of decreased bone mass, according to BMD, only in the osteopenia group. Increased levels of RANKL could describe the severity of decreased bone mass based on BMD only in the osteoporosis group. Clinical manifestations and BMD tests were still needed in the interpretation of RANKL levels and OPG levels in post-menopausal females with osteoporosis.

\section{REFERENCES}

1. Sözen, T, Özı şı k, L, Başaran, NC. An overview and management of osteoporosis. European Journal of Rheumatology, 2017; 4:46-56.

2. Mithal A, Kaur P. Osteoporosis in Asia: A call to action. Curr Osteoporos Rep, 2012; 10: 245-7.

3. Kementerian Kesehatan Republik Indonesia. Data \& kondisi penyakit osteoporosis di Indonesia. Jakarta, Pusat Data dan Informasi Kementerian Kesehatan Republik Indonesia, 2015, 2-6.

4. Sheu A, Diamond T. Bone mineral density: Testing for osteoporosis. Aust Prescr, 2016; 39: 35-91.

5. Garnero P. Biomarkers for osteoporosis management-utility in diagnosis, fracture risk prediction, and therapy monitoring. Journal of Endocrine Disorders, Mol Diag Ther, 2008; 12(3): 157-70.
6. Sennels HP, Jacobsen $\mathrm{S}$, Jensen $\mathrm{T}$, Hansen MS, Ostergaard $\mathrm{M}$, et al. Biological variation and reference intervals for circulating osteopontin, osteoprotegerin, total soluble receptor activator of nuclear factor kappa $B$ ligand and high-sensitivity $C$-reactive protein. Scand J Clin Lab Invest, 2007; 67: 821-35.

7. Khosla S. Mini review: The OPG/RANKL/RANK system. Endocrinol, 2001; 142: 5050-55.

8. Boyce BF, Xing L. Biology of RANK, RANKL, and osteoprotegerin. Arthritis Research \& Therapy, 2007; 9(S1): 1689-1697.

9. World Health Organization. Body mass index-BMI. http://www.euro.who.int/en/health-topics/ disease-prevention/nutrition/a-healthy-lifestyle/ body-mass-index-bmi. (accessed 11 Nov, 2019).

10. World Health Organization. WHO scientific group on the assessment of osteoporosis at primary health care level. Brussel-Belgium, Summary Meeting Report, 2004; 2 .

11. Elabscience $\AA$. Manual book-human sRANKL (soluble receptor activator of nuclear factor-kB ligand) ELISA Kit, $7^{\text {th }}$ Ed., USA, Elabscience ${ }^{\circledR}, 2017 ; 1-11$.

12. Elabscience ${ }^{\circledR}$. Manual book-human OPG (osteoprotegerin) ELISA kit, $7^{\text {th }}$ Ed., USA, Elabscience ${ }^{\circledR}, 2017 ; 1-11$.

13. Trofimov S, Pantsulaia I, Kobyliansky E, Livshits G. Circulating levels of receptor activator of nuclear factor-kB ligand/osteoprotegerin/ macrophage-colony stimulating factor in a presumably healthy human population. European Journal of Endocrinology, 2004; 150: 305-11.

14. Widodo H, Anisa FN, Lianti D. Relationship of physical activity, diet, and age menarche with age menopause at elderly Posyandu working area Puskesmas Pekauman Banjarmasin. Advances in Health Science Research, 2017; 6: 566-74.

15. Ahuja M. Age of menopause and determinants of menopause age: A PAN India survey by IMS. J Mid-life Health, 2016; 7: 126-31.

16. Gold EB. The timing of the age at which natural menopause occurs. Obstet Gynecol Clin N Am, 2011; 38: $425-40$.

17. Lambrinoudaki I, Tsouvalas E, Vakaki M, Kaparos G, Stamatelopoulos $\mathrm{K}$, et al. Osteoprotegerin, soluble receptor activator of nuclear factor- $\mathrm{k} B$ ligand, and subclinical atherosclerosis in children and adolescents with type 1 diabetes mellitus. International Journal of Endocrinology, 2013; 2013: 1-8.

18. Holecki M, Zahorska-Markiewicz B, Janowska J, Nieszporek T. The influence of weight loss on serum osteoprotegerin concentration in obese perimenopausal women. Obesity, 2007; 15(8): 1925-9.

19. Dimitri P, Wales JK, Bishop N. Adipokines, bone-derived factors and bone turnover in obese children; Evidence for altered fat-bone signaling resulting in reduced bone mass. Bone, 
2011; 48(2): 189-96.

20. Hsu YH, Venners SA, Terwedow HA, Feng Y, Niu $\mathrm{T}$, Li Z, et al. Relation of body composition, fat mass, and serum lipids to osteoporotic fractures and bone mineral density in Chinese men and women. Am J Clin Nutr, 2006; 83: 146-54.

21. Ahmad EA, Abbas M, Amir R, Mohammad S. The relationship between body weight, Body Mass Index (BMI) and Bone Mineral Density (BMD) of the lumbar spine and femoral neck in professional cyclist of Iran and tour de France. Iranian Journal of Health and Physical Activity, 2012; 4: 67-71.

22. Iqbal SI, Morch LS, Rosenzweig M, Dela F. The outcome of bone mineral density measurements on patients referred from general practise. Journal of Clinical Densitometry, 2005; 8: 178-82.

23. Nabipour I, Larijani B, Vahdat K, Assadi M, Jafari $\mathrm{SM}$, et al. Relationships among serum receptor of nuclear factor-JB ligand, osteoprotegerin, high-sensitivity $\mathrm{C}$-reactive protein, and bone mineral density in post-menopausal women: osteoimmunity versus osteoinflammatory. The Journal of the North American Menopause Society, 2009; 16(5): 950-5.

24. Kim SJ, Yang WG, Cho E, Park E. Relationship between weight, body mass index, and bone mineral density of lumbar spine in women. J Bone Metab, 2012; 19: 95-102.

25. Fahrleitner-Pammer A, Dobnig H, Piswanger-Soelkner, C. Osteoprotegerin serum levels in women: correlation with age, bone mass, bone turnover, and fracture status. Wien
Klin Wochenschr, 2003; 115: 291-7.

26. Mendez JP, Mejia DR, Pedraza J, Vazquez RMC, Soriano $\mathrm{R}$, et al. Bone mineral density in post-menopausal Mexican-Mestizo women with normal body mass index, overweight, or obesity. Menopause, 2013; 20: 568-72.

27. Bolotin HH. DXA in-vivo BMD methodology: An erroneous and misleading research and clinical gauge of bone mineral status, bone fragility, and bone remodeling. Bone, 2007; 41: 138-54.

28. Brown SE. Osteoporosis: Snap, crackle, and pop some pills. Northeast Florida Med, 2006; 57: 14-20.

29. Shanthi JC, McLeod W, Kennedy L, McLeod K. Osteoporosis health beliefs among younger and older men and women. Health Educ Behav, 2008; 35(5): 721-33.

30. Pietschmann P, Rauner $M$, Sipos $W$, Schindl KK. Osteoporosis: An age-related and gender-specific disease a mini-review. Gerontology, 2009; 55: 3-12.

31. Liu JM, Zhao HY, Ning G, Zhao YJ, Chen Y, et al. Relationships between the changes of serum levels of OPG and RANKL with age, menopause, bone biochemical markers and bone mineral density in Chinese women aged 20-75. Calcif Tissue Int, 2005; 76: 1-6.

32. Ginaldi L, Di Benedetto MC, De Martinis M. Osteoporosis, inflammation, and aging. Immun Ageing, 2005; 2: 14 .

33. Gallagher JC. Advances in bone biology and new treatments for bone loss. Maturitas, 2008; 60: 65-9. 